\title{
Las mujeres inmigrantes en Sevilla: salud y condiciones de trabajo. Una revisión desde la medicina social
}

\author{
María J. Río Benito \\ Manuela Álvarez Girón \\ Carmen García Gil \\ Ana Solano Parés \\ Universidad de Sevilla \\ E-mail: solano@ us.es
}

RESUMEN

La mayor parte de las mujeres inmigrantes que viven en Sevilla trabajan en el servicio doméstico. Sus quejas en los servicios de urgencias y de atención primaria, según la impresión de los profesionales sanitarios, van más allá de los síndromes y encierran toda su historia. La atención directa a estas mujeres en los servicios sociales y sanitarios, así como la gestión a medio-largo plazo de los servicios para el futuro, necesitan de una mirada integradora que contemple los movimientos migratorios, las nuevas relaciones laborales en la era de la globalización, las relaciones salud-género y la cultura, que lo atraviesa todo. Una mirada del profesional que intente comprender con la finalidad de reducir las desigualdades en salud evitables.

Palabras clave: Mujeres Trabajadoras, Inmigrantes, Salud. 


\section{LA INMIGRACIÓN EN ANDALUCÍA}

En los años ochenta, Andalucía pasó a contar con los dos rasgos que caracterizan hoy a la mayoría de países y regiones de la Unión Europea: recibir una inmigración neta y tener una fecundidad en declive. La inmigración no es un fenómeno nuevo en Andalucía, ya que en los años sesenta y setenta apareció una inmigración de personas mayores fundamentalmente en las regiones costeras de Málaga. La evolución económica y política de los países mediterráneos vecinos, de los países del África subsahariana, de Latinoamérica y del Este europeo, unido a la sectorización de nuestro mercado de trabajo, que permite coexistencia de paro y de ofertas de empleo no cubiertas, pueden crear las condiciones para la entrada creciente de trabajadores inmigrantes.

Para realizar un adecuado abordaje del complejo fenómeno de la inmigración hay que analizar la estructura económica de la sociedad receptora. Andalucía se sitúa en la posición 158 de las 160 regiones de la UE según indicadores socioeconómicos (Delgado, 1991), manteniendo en la actualidad niveles de paro muy altos. Por ello hay autores como Emma Martín (1999) que se refieren a Andalucía como semiperiferia en términos económicos. Si bien en nuestra Comunidad ha aumentado la productividad, ha sido a expensas del crecimiento de sectores relacionados con el exterior y complementarios de otras economías. Las relaciones laborales en Andalucía, como en el resto de España, se sitúan desde 1985, según Gavira (1999), en una etapa de «individualización e informalización» en que la precarización afecta a todas las capas sociales.

Los inmigrantes no son un conjunto homogéneo. Según De Prada (2001), las características de las migraciones actualmente son: Diversidad, Transnacionalización, Circularidad y Feminización. En el panorama mundial del fenómeno migratorio hay que incluir no sólo la migración llamada económica del Sur al Norte, sino también a los refugiados, los flujos entre países del Sur (que son los más importantes en número), de los países del Este de Europa, de personal cualificado de empresas transnacionales entre países del Norte, y del Norte al Sur generalmente para tareas denominadas en su conjunto como humanitarias. La circularidad es aplicada a los proyectos migratorios de ida y vuelta posibles por la mejora considerable de los medios de comunicación.

Los inmigrantes que llegan a España también son diversos. No todos llegan en pateras. Más de la mitad lo hace valiéndose del estatus de turista, estudiante o con un visado. Desde el punto de vista económico de los países receptores, uno de los problemas fundamentales que plantea la inmigración es la incorporación estructural de los extranjeros. Los dos modos más ampliamente aceptados son: la incorporación a los mercados de trabajo y, según Portes (1981), la «formación de enclaves étnicos». En el primer caso, los inmigrantes 
se incorporan al mercado de trabajo secundario, inestable, con bajos salarios y cualificación y con escasas posibilidades de promoción. Y, en el segundo, se establecen negocios propios siempre y cuando exista previamente un grupo étnico significativo culturalmente y socialmente agrupado. Habría que añadir la inserción en el trabajo precario, informal, en el que son fácilmente explotados los inmigrantes en situación irregular.

Una de las primeras dificultades con que nos encontramos a la hora de estudiar la inmigración en nuestra Comunidad es la cuantificación. Según datos oficiales del Ministerio del Interior a 31 de diciembre de 2000, en España había 938.783 extranjeros con permiso de residencia, lo que suponía un 2,5\% de la población total. De ellos, 515.955 provenían de países no incluidos en la Unión Europea. A principios de 2001, según el Instituto de Estadística de Andalucía (IEA), en nuestra Comunidad residían 138.126 extranjeros, un 15\% del total de residentes extranjeros en España. Según datos del Observatorio Europeo del Racismo y la Xenofobia del año 2000, en España se estima un número de inmigrantes irregulares de 300.000. Aplicando a esta estimación la misma proporción en Andalucía que para los regularizados (el 15\%), en nuestra Comunidad habría 45.000 inmigrantes no regularizados. Según datos de la Dirección General de la Policía relativos al año 2000, en Sevilla, del total de 9.650 extranjeros residentes, 2.409 eran mujeres adscritas al Régimen General' ${ }^{1}$.

Si bien en los años ochenta se relaciona a la mujer inmigrante con su papel de reagrupada en el proyecto migratorio de su compañero, en los noventa se reconoce su papel activo en los procesos migratorios convirtiéndose en jefas del hogar. Según estudio de loé (1997), se distinguen cinco tipos de proyectos migratorios de las mujeres: para asegurar la subsistencia del grupo familiar, solteras que buscan promoción personal, por espíritu aventurero, siguiendo el proyecto migratorio del marido, jóvenes para reunirse con familiares ya emigrados.

Las cuatro actividades laborales más frecuentes identificadas por loé (1996), que agrupan el $75 \%$ de las mujeres no comunitarias con permiso de trabajo, son el servicio doméstico $(>50 \%)$, la hostelería y restauración $(11 \%)$, el comercio $(4,4 \%)$ y las actividades industriales $(4,4 \%)$. Este perfil ocupacional difiere de forma apreciable del que presentan las mujeres autóctonas, que se emplean principalmente en actividades comerciales y de reparaciones $(20,2 \%)$, industria manufacturera $(12,5 \%)$, educación, sanidad y servicios sociales ( $10 \%$ cada una), servicios inmobiliarios, hostelería y servicio doméstico ( $7 \%$ cada una). Los procesos de deslocalización ${ }^{2}$ industrial conllevan cada vez menor necesidad de mano

\footnotetext{
1 Se refiere a los extranjeros no procedentes de los Estados miembros de la Unión Europea ni de los países a los que se aplica el régimen comunitario (Noruega, Islandia y Liechtenstein).

2 El trabajo intensivo se ha separado del capital intensivo, instalándose los procesos productivos en zonas donde la fuerza de trabajo tiene coste mínimo (Narotzky, 1988).
} 
de obra extranjera para las actividades en el Norte, puesto que los procesos de producción se exportan al Sur. Así, los inmigrantes han dejado de cumplir su papel de mano de obra industrial para pasar a cubrir los empleos en el sector servicios. La migración de mujeres del Sur al Norte responde a esa demanda creciente de mano de obra en el sector servicios para ocupar empleos generalmente abandonados por las autóctonas (servicio doméstico, cuidados asistenciales, etc.).

Carmen Gregorio (citada en loé, 1996) ha propuesto cinco ejes para analizar la exclusión de la mujer inmigrante: lógica del estado-nación, lógica de la cultura dominante, lógica de clases, lógica de género y lógica racial. Para Dolores Juliano (1993), nuestra visión peyorativa de las mujeres inmigrantes se basa en gran medida en su supuesta mayor discriminación frente a los hombres. Las políticas de inmigración tienen efectos desiguales sobre hombres y mujeres. La regularización de una mujer extranjera se realiza a partir de su condición de cónyuge de un inmigrante o de un español o ciudadano comunitario, lo que se traduce en un permiso de residencia no laboral colocando a la mujer en situación de dependencia respecto a su pareja, apartándola del mercado de trabajo y obligándola en cierto modo a trabajar en condiciones de irregularidad. Además, existen disposiciones que favorecen el acceso a la situación regular a las mujeres inmigrantes como los contingentes laborales para el servicio doméstico, lo que refuerza la feminización de ciertos colectivos.

\section{EL SERVICIO DOMÉSTICO, A MITAD DE CAMINO ENTRE LA ECONOMÍA DOMÉSTICA Y LA MONETARIA}

Las tareas domésticas ${ }^{3}$ son imprescindibles en todas las culturas para el bienestar de sus miembros, cumpliendo funciones no sólo de sustento de las necesidades básicas (alimentación, higiene y vestido), sino también afectivas al proporcionar el cuidado de lo íntimo.

Tradicionalmente, las tareas domésticas se han asignado a las mujeres, que las han realizado como un mandato de género sin plantearse por su realización nada a cambio. Según Meillassoux (1987), el contrato matrimonial legisla sobre el patrimonio pero no sobre el trabajo realizado dentro del hogar, que se considera no productivo. En las sociedades patriarcales se han separado los espacios público y privado. Las mujeres, a la vez que protagonistas del espacio privado, del hogar, ocupan un papel secundario en la sociedad. Al espacio doméstico propio de las mujeres se le otorga una valoración negativa. Las tareas domésticas no son tenidas en cuenta en las estadísticas económicas mundiales al equipa-

3 El término doméstico se ha aplicado a diferentes conceptos. Algunos autores como Narotzy (1988) diferencian entre «hogar», centrado en torno a la producción y la corresidencia; «familia», centrado en torno a la reproducción y al parentesco, y «grupo doméstico», término entre los anteriores más próximo al de hogar en ambientes campesinos. 
rar trabajo con empleo remunerado. Una de las reivindicaciones de feministas como Dalla Costa (1975) ha sido la consideración del trabajo doméstico como trabajo productivo ${ }^{4}$.

Durante mucho tiempo se pensó que con la incorporación de las mujeres al mercado de trabajo remunerado se conseguiría un mayor equilibrio entre géneros en la ocupación de los espacios público y privado, así como un mayor protagonismo social de las mujeres. Las conquistas de las mujeres a finales del siglo xx son innegables: un mayor nivel educativo y una mayor incorporación al mercado laboral y a la escena pública. En 1999 las mujeres alcanzaron los mejores porcentajes en la incorporación a la educación. Según datos publicados por el Instituto Andaluz de la Mujer (IAM), en 2001 la tasa de actividad $^{5}$ de las mujeres en Andalucía sigue siendo inferior a la de los hombres: $37 \%$ frente al $63,3 \%$. Las tasas de paro de las mujeres en nuestra Comunidad duplican las de los hombres: $30,1 \%$ frente al $16,1 \%$, ambas muy superiores a la media de España. De las mujeres que realizan una actividad extradoméstica remunerada, sólo el 56,2\% tiene un contrato formalizado. Para comprender las diferencias del trabajo desempeñado por las mujeres nos parece de gran utilidad la caracterización de éste por Narotzky (1988) de «ayuda», frente al verdadero «trabajo» desempeñado por el hombre.

Esta presencia creciente de las mujeres fuera del hogar ha determinado cambios demográficos por el retraso de la maternidad y la reducción del número de hijos. La edad media de la mujer andaluza para la maternidad son treinta años, y su nivel de fecundidad es de 1,28 hijos por mujer, por encima de la media española del 1,15 (IAM, 2001).

De Villota (1999) plantea la cara negativa de la incorporación de la mujer al trabajo remunerado, caracterizada por fenómenos como el abaratamiento de los salarios y la segmentación rápida del mercado laboral en especialidades femeninas. Una segunda fase de esta feminización del mercado laboral la describe como la desaparición del lugar de trabajo, volviendo las mujeres a realizar trabajos con base en el hogar. Dentro de las formas de trabajo doméstico (dejando a un lado las tareas que realizan sus miembros para cubrir sus necesidades), siguiendo la clasificación que realiza el Colectivo loé6 (2001), se pueden distinguir el empleo doméstico o servicio doméstico, el trabajo a domicilio que hace referencia al realizado en su mayor parte por los miembros del hogar para tareas sobre todo

\footnotetext{
4 En el análisis del sistema capitalista se ha diferenciado producción, que produce una mercancía, y reproducción. Dentro del concepto de reproducción, autores como Edholm, Harris y Young (1977) distinguen: 1) reproducción social; 2) reproducción de la fuerza de trabajo (donde se incluyen las tareas domésticas), y 3) reproducción biológica.

5 Tasa de actividad incluye a las ocupadas y desempleadas.

6 El Colectivo loé es un Gabinete de Investigaciones Sociológicas en cuyo equipo de investigación se encuentran Walter Actis, Carlos Pereda y Miguel Ángel de la Prada.
} 
de confección e industriales destinadas a la venta, y los servicios de ayuda a domicilio orientados al cuidado de ancianos y enfermos.

El servicio doméstico (SD) en nuestro país, como en otros países de nuestro entorno, se encuentra regulado de forma diferencial. El Real Decreto 1424, de 1 de agosto de 1985, regula la relación laboral «de carácter especial del hogar familiar». Hay que recordar que tan sólo un siglo antes, en 1889, se proscribió el vínculo de servidumbre en España. El carácter especial de la regulación laboral incluye, entre otros aspectos: la no obligatoriedad de contrato escrito, la posibilidad de descontar hasta un $45 \%$ del salario en concepto de manutención y alojamiento (la OIT ${ }^{7}$ recomienda que no sea superior al $20 \%$ ), la exclusión de las prestaciones del subsidio de desempleo, la prolongación hasta el vigésimo noveno día para cobrar la baja por enfermedad o accidente, y la existencia del denominado tiempo de presencia que no se considera dentro del horario laboral y en el que el trabajador puede ser requerido para la realización de tareas que no exijan mucho esfuerzo. La principal reivindicación de las trabajadoras del servicio doméstico (TSD), como se ha puesto de manifiesto en diversos foros ${ }^{8}$, es la derogación del Real Decreto que regula de manera discriminatoria su actividad laboral.

EI SD es una actividad singular realizada preferentemente por mujeres (según la Encuesta de Población Activa de 1999, en un 88\%) que se encuentra a mitad de camino entre la economía doméstica y la monetaria contigua a la del ama de casa sin el estatus de ésta. El espacio privado en que se desarrolla, lugar de control de las relaciones afectivas, el aislamiento social que condiciona y la relación de dependencia que se establece frente al empleador determinan, según Ulloa (2002), las características diferenciales del SD: invisibilidad, subordinación, discriminación y servilismo.

Según la excelente revisión que sobre el SD ha realizado el Colectivo loé (2001), el número de hogares españoles que cuentan con trabajadores del servicio doméstico (TSD) se estima entre 800.000 y 1.000 .000 . Las tres Comunidades donde se concentra la mayor parte del empleo son Andalucía (18,2\%), Cataluña (17,6\%) y Madrid (13\%). Las provincias con mayor número de TSD son Madrid y Barcelona, un $12 \%$ del total cada una, seguidas de Valencia, Vizcaya y Sevilla, que representan un $5 \%$ cada una.

Los ámbitos de lo público y de lo privado se encuentran en una estrecha relación. Las actuales políticas económicas, caracterizadas por la progresiva disminución de la partici-

7 OIT es la sigla de Organización Internacional del Trabajo.

8 En las Jornadas «Mujer y Servicio Doméstico», organizadas por Mujeres entre Mundos y celebradas en Sevilla los días 8 y 9 de febrero de 2002, se concluyó de forma unánime que cualquier avance en la situación de las TSD pasa por el cambio del Real Decreto. 
pación del Estado en los servicios de bienestar social, tienen una clara repercusión en las mujeres. Como expone Rodríguez (2002), la desintegración de estos servicios públicos deja la responsabilidad en manos de la familia, del mercado y de una emergente sociedad civil. Cuando nos referimos a la familia, en nuestra sociedad patriarcal nos referimos a las mujeres. Todavía falta mucho para lograr la corresponsabilización hombre/mujer en las tareas de dentro del hogar, las mujeres son en su mayor parte las que continúan realizando las tareas del hogar independientemente de si realizan un trabajo fuera del domicilio y las que ejercen de cuidadoras. Los hombres que más cooperan en casa son los de un estatus y nivel de estudios elevados. Desde el mercado se ofrecen servicios de bienestar, entre los que se incluyen el servicio doméstico y la ayuda a domicilio, a los que sólo pueden acceder las personas con mejor situación económica. De este modo surge un nuevo desequilibrio entre las corresponsabilidades en el hogar, en este caso de género mujer/mujer, ya que las mujeres con mayor cualificación laboral y académica son las que menos se ocupan de las tareas domésticas y las que se encuentran en peor situación socioeconómica las que acceden al servicio doméstico.

Se trata, pues, de una profesión de emergencia a la que se accede generalmente en situación de necesidad, bien mujeres jóvenes sin cualificación, como primer empleo en mujeres maduras y como puerta de entrada en mujeres inmigrantes. Cada persona actúa y decide en una situación histórica determinada. Según Encuesta loé ${ }^{9}$ a trabajadores del servicio doméstico, las trabajadoras extranjeras siguen siendo minoría en el conjunto del sector del SD (11,9\%), pero representan entre el 46 y el $60 \%$ en el trabajo interno, frente a un $10 \%$ en el trabajo externo fijo y un $5 \%$ en el de asistencia por horas. Según datos ofrecidos por el Instituto de la Mujer de Andalucía ${ }^{10}$, en el año 2000 el número de mujeres dadas de alta en la Seguridad Social en Andalucía en el Régimen Especial de Empleadas del Hogar era de 13.750 , de las cuales un $18 \%$ eran extranjeras. Se estima que del total de TSD sólo una de cada cuatro está dada de alta en la SS. Con la incorporación al sector del SD de mujeres inmigrantes han aumentado los cotizantes a la SS, pero a la vez se está precarizando más el sector por la gran oferta.

\section{MEDICINA SOCIAL. SALUD Y GÉNERO}

En el grupo de mujeres inmigrantes que trabajan en el servicio doméstico se identifican, como expusimos con anterioridad, varios criterios de exclusión social de los que se deriva-

\footnotetext{
9 Encuesta loé a trabajadores del servicio doméstico. Datos publicados en Mujer, Inmigración y Trabajo, Ministerio de Trabajo y Asuntos Sociales, Madrid, 2001

10 Datos ofrecidos por doña Araceli Rubio Román, Directora de Instituto Andaluz de la Mujer, en las Jornadas «Mujer y Servicio Doméstico», organizadas por la Asociación de Mujeres entre Mundos y celebradas en Sevilla, 8 y 9 de febrero de 2002.
} 
rían, según los planteamientos de la medicina social actualmente aceptados de forma general, desigualdades en salud. Según la Organización Mundial de la Salud (OMS), el término desigualdades en salud hace referencia a las diferencias innecesarias, evitables e injustas.

El concepto de salud, y más concretamente el de salud mental, que vamos a considerar es el que propone la medicina social, que reconoce que las circunstancias psicológicas y sociales de las personas determinan de forma seria la salud a largo plazo. Uno de los conceptos clave en este abordaje de la salud es el de privación relativa, sobre todo referido al tema de la pobreza en los países de Europa, ya que el daño sobre la salud no es explicable sólo por las faltas materiales, sino por los problemas psicológicos y sociales resultantes de vivir en pobreza relativa. De aquí surge el término exclusión social, que incluye a los inmigrantes más pobres y de forma amplia a las mujeres. Las situaciones de riesgo para la salud identificadas por la epidemiología social son la falta de apoyo social, el estrés crónico laboral, la hostilidad y la desesperanza, entre otras. Para algunos autores como House (2002), es necesario integrar estas situaciones de riesgo individuales en el contexto de las fuerzas macrosociales para una adecuada comprensión.

Revisamos a continuación los diferentes aspectos de la salud de las mujeres inmigrantes que trabajan en el servicio doméstico desde la perspectiva de la medicina social.

Las relaciones salud-género están siendo objeto de debate y de estudio en las dos últimas décadas. El interés creciente en este tema ha sido fomentado, siguiendo a Hunt (1999), por «una segunda corriente feminista y por una epidemiología que estudia las desigualdades en salud». La salud, entendida en un sentido amplio como bienestar, como recurso positivo que permite la autonomía, la entendemos como un constructo cambiante con la cultura y el momento histórico. El concepto género es para muchos autores un constructo analítico fundamentado en la organización social de los sexos. Algunos autores como Scott (1988) incluyen en el concepto de género los aspectos de relación, poder, cultura, moral y la identidad subjetiva del individuo. De acuerdo con Rohlfs y colaboradores (2000), «la salud de mujeres y hombres es diferente y desigual. Diferente porque hay factores biológicos que se manifiestan de forma diferente en la salud y en los riesgos de enfermar. Desigual porque hay otros factores, explicados en parte por el género, que afectan de una manera injusta la salud de las personas».

La mayoría de las sociedades no miden el impacto en la salud del trabajo reproductivo de cuidados, crianza o trabajo doméstico que realizan las mujeres. Sin embargo, la sobrecarga de roles de cuidado parece tener un impacto negativo en la salud. En el caso del trabajo productivo llevado a cabo por las mujeres, siguiendo a Rohlfs y colaboradores (2000), las influencias sobre la salud van a depender del tipo de ocupación, de las condiciones laborales y del equilibrio entre los roles productivos y reproductivos. Estudios realizados en 
nuestro medio (Artazcoz, 2001) ponen de manifiesto el efecto protector sobre la salud de las mujeres de disponer de una persona contratada para el servicio doméstico.

Compartiendo plenamente los criterios de las Mujeres Feministas Negras y de las Mujeres Feministas del Tercer Mundo (Mohanty, 1990), es importante reconocer la opresión con sus caras múltiples de género, raza y clase que determinan en gran medida el pensamiento y las prácticas de la salud pública.

A la enfermedad mental, como al resto de enfermedades, se le reconoce un origen multifactorial, biológico, psicológico y social. Las diferencias existentes en la distribución de las enfermedades mentales por sexo se han atribuido a lo largo de la historia tanto a diferencias cerebrales, hormonales como de los mecanismos intrapsíquicos característicos de cada sexo, sin que existan datos concluyentes en la literatura. Cada vez existe mayor consenso en el papel del estrés psicosocial en la incidencia de un trastorno emocional. Colten y Gore (1984) proponen una ecuación que resume el modelo multifactorial para la enfermedad psíquica. Estarían directamente relacionados con la aparición de un trastorno emocional el estrés y la vulnerabilidad (factores de riesgo), e inversamente relacionados las capacidades de afrontamiento, el soporte social y la autoestima (factores protectores).

Montero (1999), al referirse a las hipótesis psicosociales de la depresión en la mujer, plantea que para muchas mujeres constituiría una respuesta al «dilema de vivir» frente a las exigencias del doble rol laboral y familiar. Considerando la depresión como la respuesta a una pérdida, esta misma autora destaca la pérdida de relaciones interpersonales en las mujeres frente a la pérdida de objetivos en el hombre. Távora ${ }^{11}$ (2000) explica la enfermedad psíquica de las mujeres como un conflicto en los deseos con una imposibilidad de compaginar la vida laboral y doméstica (en las independientes desde un punto de vista económico y con alto nivel educacional) o como un desconocimiento de sus propios deseos, usando como móviles de vida los deseos de los otros (en las mujeres con bajo nivel educacional y dedicadas exclusivamente a las tareas domésticas). En la misma línea, Levinton $^{12}$ (2002) señala como principales conflictos intrapsíquicos en las mujeres la lucha entre el deseo de ser buenas madres y de ser eficaces en su rol laboral, entre su deseos sexuales y sus creencias morales, y entre el reconocimiento de la propia rabia y el ideal de ser buenas. Esta misma autora plantea la vulnerabilidad para la salud mental de las mujeres como la coincidencia de varias de las situaciones siguientes: el matrimonio, las tareas domésticas características del ama de casa, el cuidado de tres o más niños pequeños, carecer de redes de apoyo, y la falta de intimidad y confidencialidad con su pareja.

11 Ana Távora es psiquiatra.

12 Nora Levinton es psicoanalista. 
La medicina social reconoce actualmente que las circunstancias psicológicas y sociales de las personas pueden dañar de forma seria su salud a largo plazo. Brunner (1999) identifica como factores psicosociales que pueden influir de forma negativa sobre la salud mental y física la ansiedad crónica, la baja autoestima, el aislamiento social y la falta de control sobre el trabajo. El poder de estos factores psicosociales sobre la salud tiene un sentido biológico, según el mismo autor, ya que la respuesta de estrés biológico activada de forma muy frecuente y prolongada puede llevar consigo múltiples costes en la salud, entre los que se incluyen: depresión, susceptibilidad aumentada para la infección, diabetes, hipertensión e hipercolesterolemia, con el aumento de riesgo para la cardiopatía isquémica y para la patología vascular cerebral. Los mecanismos de protección frente al cúmulo de desventajas psicosociales en la vida de una persona constituyen un área emergente de investigación en salud pública. Esta perspectiva de «curso vital» en la salud, según Blane (1999), identifica en el estatus biológico de una persona un marcador de su posición social en el pasado. Los procesos sociales interactúan con la salud en diferentes modos. Uno de ellos es la continuidad de circunstancias sociales desde la clase social de los padres y de las condiciones socioeconómicas en la infancia y la adolescencia y, eventualmente, la posición socioeconómica del adulto. Otro mecanismo sería la movilidad social hacia otra clase con diferentes ventajas y desventajas en salud. Un tercer mecanismo de interacción entre salud y factores sociales es la protección social, que toma mayor relevancia cuando las condiciones externas son más hostiles.

Esta perspectiva de «curso vital» en salud tiene implicaciones en las políticas sociales. La adversidad no está distribuida de forma randomizada, sino que tiende a formar clusters y a acumularse en los grupos más vulnerables y con más desventajas sociales y en salud. Una política social moderna que tuviera en cuenta estos principios de la medicina social debería, según Blane (1999): 1) identificar los individuos de riesgo, y 2) poner en marcha intervenciones efectivas que no sólo se basaran en redes de seguridad para prevenir el cúmulo de desventajas, sino también en «trampolines» que repararan el daño anterior.

Uno de los campos de investigación más reciente es el que considera la relación salud y trabajo más allá de las tradicionales enfermedades ocupacionales (Shilling, 1989). Se reconoce actualmente la importancia de los factores psicosociales en el lugar de trabajo en el gradiente social en la salud. Los diferentes modelos desarrollados para medir el estrés en el trabajo, como el de demanda-control (Karasek y Theorell, 1990) y el disbalance esfuerzo-premio (Siegrist, 1996), resultan útiles a la hora de analizar la salud de las mujeres inmigrantes que trabajan en el servicio doméstico. Karasek (1979) formuló un concepto de estrés en el trabajo bidimensional en que participaban un alto nivel de demandas psicológicas al trabajador junto a un bajo nivel de decisión y de utilización de habilidades por su parte. Este modelo se ha modificado con la inclusión del apoyo social en el trabajo. 
El modelo del disbalance esfuerzo-premio, a diferencia del anterior, que hace hincapié en características ambientales del trabajo, se centra en el desequilibrio a nivel macroeconómico entre los costes laborales y los beneficios entendidos como beneficios monetarios, en la autoestima, en las oportunidades en la carrera profesional y en la seguridad en el trabajo (Marmot, 1999).

Existen al menos cuatro razones, según Marmot (1999), para explicar la centralidad del trabajo y la ocupación en las sociedades industrializadas: el trabajo como requisito para ingresos continuos, formación para un trabajo y obtención del estatus ocupacional como los objetivos más importantes de la socialización, la ocupación como criterio más importante de la estratificación social y, finalmente, el trabajo como ocupación que absorbe mayor tiempo en la vida adulta. El trabajo en la era de la globalización ha adquirido características diferenciales, entre las que destaca la flexibilidad. Sennett (2000) analiza de forma magistral la pérdida de control sobre la propia vida que determina una vida laboral plagada de cambios. El orden del nuevo capitalismo es definido por este autor como en red, frente a la estructura piramidal propia de la anterior organización del trabajo, caracterizada por la estabilidad. Sennett señala al capitalismo flexible en la raíz de la destrucción del carácter, que entiende como «un aspecto duradero de nuestra experiencia emocional, que se expresa por la lealtad y el compromiso mutuo, bien a través de la búsqueda de objetivos a largo plazo, bien por la práctica de postergar la gratificación en función de un objetivo futuro». Plantea la dificultad que entraña el mantenimiento de un «valor duradero» en las personas en una «sociedad centrada en lo inmediato».

Para entender las nuevas reglas laborales y su relación con la salud retomamos ahora el término globalización. La OMS, en su revisión monográfica sobre Globalización y Salud, del año 2001, en un sentido práctico considera la globalización como «el proceso por el que las políticas nacionales e internacionales promocionan la desregulación de los mercados domésticos y la liberalización». Los efectos atribuidos a la globalización sobre la salud pública son diversos. Dollar (2001) identifica como mecanismos para explicar las implicaciones positivas sobre la salud de la globalización la diseminación de conocimientos y el aumento de ingresos en los países menos desarrollados por medio, entre otros, de la emigración exterior. $Y$ como efectos adversos destaca la diseminación de enfermedades y la inseguridad alimentaria. De mayor interés para el tema que nos ocupa nos parecen las reflexiones de Cornia (2001) en esta revisión sobre el impacto negativo sobre la salud infantil de la participación laboral de las mujeres sin un desarrollo paralelo institucional para el cuidado de los niños y niñas. Así como las que dedica al papel que juegan el desempleo, la inseguridad laboral y las desigualdades destructoras de la cohesión social en los trastornos relacionados con el estrés, donde incluye desde trastornos mentales, alcoholismo, violen- 
cia doméstica, suicidio, hasta enfermedades cardiovasculares. La salud de las mujeres inmigrantes que llegan a nuestro país para trabajar en el servicio doméstico se vería influida por los efectos positivos y negativos de la globalización.

El apoyo social se ha definido como «los recursos proporcionados por otras personas» (Cohen y Syme, 1985). También se ha interpretado como «la información que lleva a un sujeto a creer que es cuidado y querido, que es estimado y valorado y que pertenece a una red social de comunicación y obligación mutua» (Cobb, 1976). Las redes sociales se refieren a los contactos sociales de un grupo de personas que se pueden describir en términos de número de contactos y frecuencia. La ventaja de las medidas de redes sociales reside en su facilidad; en contrapartida, no proporcionan ninguna indicación sobre la calidad de la interacción en los contactos. Un análisis más rico se puede conseguir examinando la calidad del apoyo, que se puede dividir en emocional y práctico o instrumental. Se han descrito dos tipos de mecanismos de acción para el apoyo social sobre la salud, que, para Stansfeld (1999), son uno directo y otro de efecto amortiguador. Existe un consenso sobre el valor del soporte emocional sobre los efectos de los acontecimientos vitales y sobre las patologías psiquiátricas menores (Kessler y McLeod, 1985). Se ha encontrado que el apoyo emocional por la persona más cercana es un predictor de buena salud mental en el hombre (Stansfeld et al., 1998b), mientras que en la mujer se ha visto beneficioso sobre la salud mental el apoyo brindado por al menos las cuatro personas más cercanas (Fuhrer et al., 1999).

Los procesos que llevan a la exclusión social en Europa son complejos. Según White (1998), incluyen cambios económicos (aumento del desempleo y de la inseguridad en el empleo), cambios demográficos (aumento de la proporción de hogares con un solo miembro, familias monoparentales y personas mayores), cambios en los regímenes de bienestar social (con recortes y privatizaciones de los bienes públicos) y procesos de segregación (estigmatización y marginalización de minorías). El mismo autor diferencia cuatro aspectos de la exclusión social: por medio de las leyes, por el fracaso para ofrecer beneficios sociales a grupos con necesidades especiales, por la imposibilidad de formar parte del sector productivo de la sociedad, y por la exclusión económica que priva de las experiencias de consumo de la vida diaria.

\section{INMIGRACIÓNY SALUD. PSIQUIATRÍA TRANSCULTURAL}

Al ser la inmigración en España y en Andalucía un fenómeno relativamente reciente se cuenta con datos tan sólo parciales acerca de la salud de los inmigrantes con los que convivimos. La información en nuestra Comunidad procede fundamentalmente de las 
ONGs que atienden a los inmigrantes, especialmente de las firmantes del Convenio de Colaboración en Materia de Salud Pública para el Colectivo de Inmigrantes con la Consejería de Salud de la Junta de Andalucía ${ }^{13}$. Otra fuente interesante procede del Hospital de Poniente almeriense, al contar esta comarca con un 15-17\% de población extranjera. De los datos obtenidos de las diversas fuentes podemos sacar como primera conclusión que los principales problemas de salud de los inmigrantes no son las enfermedades tropicales. Las patologías atendidas en el Servicio de Urgencias del Hospital de Poniente, según Huer$\operatorname{tas}^{14}$ (2002), entre los inmigrantes son en porcentajes las mismas que en la población autóctona. Los principales problemas detectados, según Chamizo ${ }^{15}$ (2002), en la salud de los inmigrantes son las dificultades de acceso al sistema sanitario (mediadas en gran medida por las diferencias culturales y lingüísticas); la enfermedad más grave es el desarraigo; y el principal determinante de la salud de los inmigrantes, la situación socioeconómica desfavorable. De aquí que la inmigración económica no se pueda considerar un problema de salud pública, como pueden pretender voces alarmistas que en el fondo esconden un discurso racista, sino un fenómeno vital estresante secundario a un multiduelo al que se añaden unas condiciones de vida en muchos casos desfavorables.

Frente a una realidad de la que desconocemos casi todo se han desarrollado, según González Galiana ${ }^{16}$ (2002), lo que ella denomina «los nuevos tópicos de la inmigración» al hablar de salud. Entre éstos incluye el uso prominente del término inmigración en singular, que nos trae a la cabeza un varón de origen magrebí; proponiendo el uso del término en plural, que refleja mejor la realidad diversa a la que alude. Otros de los tópicos que señala es el abordaje preeminente de salud como ausencia de enfermedad, y el de que todos los inmigrantes están fundamentalmente sanos, olvidando que «en situaciones de precariedad patologías leves pueden agravarse».

Para la obtención de información sobre las condiciones de salud de la población inmigrante en nuestra Comunidad podemos recurrir, siguiendo a Ugalde (1997), a la morbilidad atendida, observada, percibida y clínica. No existen datos sobre morbilidad atendida en atención primaria. La Sociedad Española de Medicina Familiar y Comunitaria (SEMFYC) (2002) identifica como dificultades en la accesibilidad al sistema sanitario debidas al propio

\footnotetext{
${ }^{13}$ El Convenio de Colaboración en Materia de Salud Pública para el Colectivo de Inmigrantes fue suscrito el 19 de marzo de 1999 entre la Consejería de Salud de la Junta de Andalucía y la Federación Andalucía Acoge, la Asociación Médicos del Mundo, la Cruz Roja Española en Andalucía y la Fundación Progreso y Salud; con la adhesión el 8 de octubre de 1999 de los sindicatos CC.OO. y UGT, y el 14 de noviembre de 2001 de la Asociación Cardijn-Tartessos.

14 Manuel Huertas es el Gerente del Hospital de Poniente de Almería.

15 José Chamizo es el Defensor del Pueblo andaluz.

16 Teresa González Galiana es la responsable médica de Médicos del Mundo en Andalucía.
} 
sistema: las actitudes del personal sanitario, la presencia de redes paralelas de atención (ONGs) y «el entramado administrativo»; y basadas en las propias características de la población: las diferencias lingüísticas y culturales, el temor por su situación irregular y las condiciones sociolaborales.

En Sevilla, la ONG firmante del Convenio que más inmigrantes recibe para la gestión de la tarjeta sanitaria provisional que permite el acceso al sistema sanitario público es Médicos del Mundo. En el año 2001 recibió 3.445 usuarios, de los que la mayoría, 3.105, como era de esperar, eran primeras visitas. De estos usuarios nuevos, 1.787 fueron mujeres, de las que 606 trabajaban en el servicio domético y 877 estaban sin ocupación. En este período se realizaron 2.060 atenciones sanitarias, de las que 58 eran de salud mental, siendo las 2.622 restantes hasta completar las 4.682 realizadas a lo largo del año de tipo social. Los cinco grupos de patologías detectadas son: enfermedades infecciosas (17,33\%), enfermedades del aparato digestivo (12,67\%), enfermedades del sistema nervioso y de los órganos de los sentidos $(11,81 \%)$, enfermedades del sistema osteomuscular y tejido conectivo $(11,55 \%)$, y trastornos mentales $(8,53 \%)$.

En Andalucía, de forma mayoritaria, desde la Administración sanitaria, desde las organizaciones científicas, desde las ONGs, la hipótesis de trabajo a la hora de abordar la salud de los inmigrantes es que la salud es un derecho humano. No se considera al grupo heterogéneo de inmigrantes como un grupo de riesgo. Se acepta en principio el «efecto emigrante sano», considerando que la mayoría de inmigrantes económicos tienen una salud normal que se ve afectada por factores derivados de su nueva situación. Entre éstos, según Ballesteros (1997), referido a inmigrantes en Roma, se encontrarían: falta de trabajo, ocupaciones de alto riesgo, ausencia de apoyo familiar, cambios en el clima y en la alimentación, riesgo de hábitos perniciosos (alcohol, drogas) y de delincuencia. Todo ello derivaría a lo que el autor resume en las patologías del malestar y en las enfermedades de la pobreza (como la tuberculosis).

Consideramos a continuación dentro de las patologías del malestar las que provocan sufrimiento psíquico desde la óptica de la psiquiatría transcultural. La psiquiatría contemporánea reconoce, siguiendo a Alarcón (2000), la influencia de factores culturales en la etiopatogenésis, diagnóstico y tratamiento de las enfermedades mentales. Esto se debe a que los psiquiatras utilizan, según Brody (1969), referentes y contextos socioculturales para evaluar y explicar la manera de pensar, sentir y actuar de los seres humanos, expresada tanto como experiencia subjetiva cuanto como conducta observable.

Cultura es para la sociedad lo que la memoria para los individuos (Kluckhohn, 1954). Es la parte del ambiente hecha por los humanos (Herskovits, 1955). «Es el conjunto de normas, valores y significados utilizados por miembros de una sociedad determinada en la cons- 
trucción de su singular percepción del mundo y de los otros grupos que los rodean. Tales normas abarcan desde elementos materiales tales como dieta, vivienda, desarrollo tecnológico o filosofía económica hasta lenguaje, religión, principios éticos, mitos, arte, rituales de convivencia social, expresión no verbal de pensamientos y emociones, consideraciones de género y estilos de vida. No se trata de una noción estática sino en constante cambio a lo largo de una permanente transmisión intergeneracional» (Alarcón, 2000).

Pike (1997), a partir de los términos fonemics (sonidos que se dan en una sola lengua) y fonetics (sonidos que ocurren en todas las lenguas), acuñó los términos aspectos emics y etics de la cultura: etics refleja constructos que se aplican a los fenómenos que ocurren en todas las culturas, y emics son aquellos que ocurren en una sola cultura.

Si bien existe un aceptable grado de acuerdo en la definición operativa de psiquiatría cultural, el debate doctrinario, como plantea Alarcón (2000), tiene que ver con su papel (descriptivo vs. comparativo), su énfasis (explicativo o interpretativo), su extensión (ciencia de la conducta social morbosa vs. sociología de la enfermedad mental) o su función (teórica vs. técnico-clínica).

«La psiquiatría cultural estudia la influencia que la cultura ejerce sobre procesos cognitivos, afectivos, comunicativos, psicofisiológicos y conductuales, al tiempo que intenta comprender también su rol en la actividad interpersonal del paciente, en particular la que tiene que ver con patrones de enfermedad y de respuesta al estrés. Evalúa asimismo el papel de factores biológicos (en tanto que activados por factores culturales) en salud y enfermedad mental y de la compleja relación entre psicoterapia y cultura» (Alarcón, 2000). Para Alarcón (2000), las funciones de la cultura en la psicopatología clínica son cinco: instrumento interpretativo y explicativo, agente patogénico y patoplástico, factor diagnóstico y nosológico, papel terapéutico y protector, y componente del manejo y provisión de servicios.

El conocimiento de la salud mental de los inmigrantes en nuestra Comunidad es un nuevo reto para la sociedad. Las relaciones entre los trastornos psicopatológicos y la migración, según Márquez (2000), se explican desde diferentes modelos, siendo uno de los más aceptados el que considera la inmigración como un multiduelo. Diversos autores, entre los que se encuentra Atxotegui (2000), consideran la migración como un acontecimiento de la vida que supone una situación de cambio con «siete duelos específicos: por la familia y amigos, por la lengua, por la cultura, por la tierra, por el estatus, por el contacto con el grupo étnico, y por los riesgos físicos».

Para Berry (1991), las relaciones entre estrés y aculturación, entendida ésta como la puesta en contacto de dos culturas, dependen de cinco factores: 1) el modo de aculturación; 
2) la fase de aculturación; 3) el tipo de sociedad receptora; 4) las características del grupo de inmigrantes, y 5) las características personales del inmigrante. Se distinguen cuatro modos principales de aculturación según las relaciones del inmigrante con las culturas de origen y receptora: integración, asimilación, separación y marginación. La integración hace referencia a una forma adaptada de relación con ambas culturas, siendo la que se asocia a menos trastornos psiquiátricos. En la asimilación se adopta la cultura del país receptor, rechazando la cultura de origen. La separación niega la cultura receptora, facilitando el establecimiento de guetos. La marginación determina el mayor número de trastornos psiquiátricos al rechazar los individuos ambas culturas. Las fases de la aculturación que siguen una evolución en el tiempo son: contacto, conflicto, crisis y adaptación. En la actualidad, según García-Campayo (2002), se piensa que el estrés aumenta en el emigrante en los dos primeros años. La sociedad receptora puede ser multicultural o asimilacionista. Según Álvarez (2002), multiculturalismo es «una manera de regular los pluralismos de los individuos y de los grupos y la aceptación de sus reivindicaciones, es el reconocimiento en un espacio público de las diferencias». Jiménez (2002) plantea el multicultarismo como «un primer intento de gestionar la pluralidad» y plantea como una segunda modalidad el interculturalismo, que frente al primero, que sitúa el énfasis en las diferencias, se centra en las semejanzas.

\section{A MODO DE RESUMEN}

Las mujeres inmigrantes que viven en Sevilla trabajan, recogiendo el testimonio de una de ellas, en tareas de esfuerzo físico o en situaciones de dependencia; refiriéndose a los trabajos como externas o internas, respectivamente, en el servicio doméstico. Realizan estos trabajos por ser los únicos que se les ofrecen, sustituyendo en sus funciones a las mujeres españolas, que ven en ellas la única alternativa para la incorporación al mundo laboral ante los escasos recursos sociales proporcionados por el Estado.

Si bien estas mujeres son pocas y muy diversas, representan una población de interés en salud pública fundamentalmente por sufrir varios criterios de exclusión social que determinan desigualdades en salud evitables. Desempeñan frecuentemente tareas domésticas y de cuidado en jornadas muy largas que les determinan agotamiento y aislamiento social. El cumplimiento de este trabajo les reporta ingresos económicos con los que generalmente mantienen a sus familiares en los países de origen, pero les priva de la realización profesional y les aleja de sus hijos, que quedan al cuidado de abuelas o tías.

La salud mental de las mujeres inmigrantes que trabajan en el servicio doméstico en nuestra ciudad se ve influida, aparte de por factores personales, por el grado de apoyo, por las condiciones laborales y por la posibilidad de compaginar su vida laboral y familiar. La medi- 
cina y la psiquiatría transcultural tendrán que estar atentas a los diferentes modelos de enfermar según las culturas para poder ser útiles a esta población. De todos modos, nos parece básico que atiendan a las relaciones dignidad, derechos humanos y salud que intentarían resolver las cuestiones políticas de las necesidades de estas mujeres y de los diversos significados que para ellas pueden tener los términos polisémicos dignidad y derechos humanos. Para ello va a resultar imprescindible el desarrollo práctico de una sociedad multicultural de ciudadanos de pleno derecho.

\section{BIBLIOGRAFÍA}

ALARCÓN, R. D. (2000): «Pasado, presente y futuro de la Psiquiatría Cultural», Monografías de Psiquiatría (4): $1-8$.

ÁLVAREZ, I. (2002): Multiculturalismo, ponencia presentada en el Foro sobre Inmigración organizado por la Asociación Pro-Derechos Humanos de Andalucía y celebrado en Cádiz los días 7 y 8 de junio.

AMABLE, M., y BENACH, J. (2000): «La precariedad laboral. ¿Un nuevo problema de salud pública?», Gaceta Sanitaria, 14 (6): 418-421.

ARTAZCOZ, L., y cols. (2001): «Trabajo doméstico, género y salud en población ocupada», Gaceta Sanitaria, 15: 150-153.

ATXOTEGUI, J. (2000): «Los duelos de la migración: una aproximación psicopatológica y psicosocial», en Medicina y Cultura. Estudios entre la Antropología y la Medicina, Perdiguero y Comelles (eds.), Bellaterra, Barcelona.

BALLESTEROS, J. M. (1997): “El Proyecto Cáritas/ASFR de Atención de Salud en Roma», en Inmigración, Salud y Políticas Sociales, O. Solas y A. Ugalde (eds.), Escuela Andaluza de Salud Pública, Serie Monografías, n. ${ }^{\circ} 13$, Granada.

BERRY, J. W. (1991): Managing the Process of Acculturation for Problem Prevention, in Mental Health Services for Refugees, National Institute of Mental Health, Washington, DC.

BETTCHER, D., y LEE, K. (2002): «Globalization and Public Health», J. Epidemiol. Community Health, 56: 8-17.

BLANE, D. (1999): «The Life Course, The Social Gradient and Health», en Social Determinants of Health, M. Marmot y R. Wilkinson (eds.), Oxford University Press, New York.

BONELLI, E. (2001): Tráfico e inmigración de mujeres en España. Colombianas y ecuatorianas en los servicios domésticos y sexuales, ACSUR-Las Segovias, Madrid.

BORRELL, C., y cols. (1999): «Social Inequalities in Perceived Heatlh and the Use of Health Services in a Southern European Urban Area», International Journal of Health Services, 29 (4): 743-764.

BRODY, E. B. (1969): «The Concerns of Transcultural Psychiatry», Am. J. Psychiatry, 125: 179-182.

BRUGULAT, P.; SÉCULL, E., y FUSTÉ, J. (2001): «Estado de salud y género en Cataluña. Una aproximación a través de las fuentes de información disponibles», Gaceta Sanitaria, 15 (1): 54-60.

BRUNNER, E. (1999): “Social Organization, Stress and Health», en Social Determinants of Health, M. Marmot y R. Wilkinson (eds.), Oxford University Press, New York. 
CARRASCO, C. (1991): El trabajo doméstico y la reproducción social, Instituto de la Mujer, Madrid.

CASTILLO, S., y MAZARRASA, L. (1999): Guía de autocuidados para la mujeres inmigrantes, Escuela Nacional de Sanidad, Madrid.

CHAMIZO, J. (2002): El derecho a la protección de la salud de los inmigrantes, conferencia inaugural de la I Jornada de Coordinación de Atención Sanitaria a Inmigrantes en Andalucía, organizada por la Fundación Progreso y Salud y celebrada en Sevilla el 20 de marzo.

COBB, S. (1976): «Social Support as a Moderator of Life Stress», Psychosom. Med., 38: 300-313.

COHEN, S., y SYME, S. L. (1985): Social Support and Health, Academic Press, London.

COLECTIVO CATRE (2001): Mujeres inmigrantes en Sevilla: nuevas catalizadoras de integración, Delegación de la Mujer, Ayuntamiento de Sevilla, Sevilla.

COLECTIVO IOÉ (1990): El servicio doméstico en España. Entre el trabajo invisible y la economía sumergida. Informe de investigación, JOC-E, Madrid.

- (1996): Procesos de inserción y exclusión social de las mujeres inmigrantes no comunitarias, Instituto de la Mujer, Madrid.

- (1997): Búsqueda bibliográfica sobre inserción profesional de las mujeres inmigrantes en varios países de la UE. Informe sobre España, Madrid.

- (1999): Inmigrantes, trabajadores, ciudadanos. Una visión de las migraciones desde España, Madrid.

- (2001): Mujer, inmigración y trabajo, Ministerio de Trabajo y Asuntos Sociales, Madrid.

COLTEN, M. E., y GORE, S. (1984): Adolescent Stress: Causes and Consequences, Aldine de Grupter, New York.

COLLINS, P. H. (1991): Black Feminist Thought: Knowledge, Consciousness, and the Politics of Empowerment, Routledge, New York.

COMISIÓN INTERDEPARTAMENTAL DE POLÍTICAS MIGRATORIAS (2001): Documento marco: Plan Integral para la Inmigración en Andalucía, Consejería de Gobernación, Junta de Andalucía, Sevilla.

CORNIA, G. A. (2001): «Globalization and Health: Results and Options», Bulletin of the World Health Organization, 79 (9): 834-841.

DALLA COSTA, M., y JAMES, S. (1975): El poder de la mujer y la subversión de la comunidad, Siglo XXI, Madrid.

DELGADO, M., y otros (1991): «La dinámica económica en Andalucía», en Interdependencia e identidad andaluza ante la integración europea, Centro de Estudios Ramón Areces, Madrid.

DE ONIA, M., y VILLAR, J. (1992): La mujer y la salud en España, Ministerio de Asuntos Sociales, Instituto de la Mujer, Madrid.

DE PRADA, M. A. (2001): Perspectivas y desafíos de las migraciones, conferencia presentada en la X Asamblea de Andalucía Acoge, Sevilla, marzo.

DE VILLOTA, P. (1999): Globalización y género, Síntesis, Madrid.

DOLLAR, D. (2001): «Is Globalization Good for your Health?», J. Epidemiol. Community Health, 56: 8-17.

DURÁN, M. a A. (1986): La jornada interminable, Icaria, Barcelona.

EDHOLM, F.; HARRIS, O., y YOUNG, K. (1977): “Conceptualizing Women», Critique of Anthropology, vol. 3. 
ESTEBAN, M. L. (1993): «La salud de las mujeres: nuevas preguntas para nuevas perspectivas», en Sistemas de Género y Construcción (Deconstrucción) de la Desigualdad, Díez y Maqueira (coords.), VI Congreso de Antropología, Tenerife.

FUHRER, R., et al. (1999): «Gender, Social Relations and Mental Health: Prospective Findings from an Occupational Cohort (Whitehall II Study)», Soc. Sci. Med., 48: 77-87.

FUNDACIÓN PROGRESO Y SALUD (1999): Convenio de Colaboración en Materia de Salud Pública para el Colectivo de Inmigrantes, Sevilla.

- (2002): Memoria Convenio de Salud para Inmigrantes 2001 y Propuesta de Programa 2002-2003, Sevilla.

GARCÍA CALVENTE, M. (1999): Cuidados y cuidadores en el sistema informal de salud, EASP, Serie Monografías, n. ${ }^{\circ} 27$, Granada.

GARCÍA-CAMPAYO, J., y SANZ CARRILLO, C. (2002): «Salud mental en inmigrantes: el nuevo desafío», Med. Clin., 118 (5): 187-191.

GAVIRA, L. (2000): «Mujeres, trabajo y dispositivos de asesoramiento», en La mujer en el mercado de trabajo, P. Rodríguez y C. Román (eds.), Consejería de Trabajo e Industria de la Junta de Andalucía y Fondo Social Europeo, Publ. IDR, Sevilla.

- (2002): Andalucía sobreviviendo en la globalización, MERGLABUM, Sevilla.

GONZÁLEZ, E., y COMELLES, J. M. (2000): Psiquiatría transcultural, Asociación Española de Neuropsiquiatría, Madrid.

GONZÁLEZ GALIANA, T. (2002): Los nuevos tópicos, ponencia presentada en la I Jornada de Coordinación de Atención Sanitaria a Inmigrantes en Andalucía, organizada por la Fundación Progreso y Salud y celebrada en Sevilla el 20 de marzo.

HERSKOVITS, M. J. (1955): Cultural Anthropology, Knopf, New York.

HOUSE, J. S. (2002): «Understanding Social Factors and Inequalities in Health: $20^{\text {th }}$ Century Progress and $21^{\text {st }}$ Century Prospects», J. Health Soc. Behav., 43 (2): 125-42.

HUERTAS, M. (2002): Memoria de la atención a inmigrantes en el Hospital del Poniente en el año 2001, ponencia presentada en la I Jornada de Coordinación de Atención Sanitaria a Inmigrantes en Andalucía, organizada por la Fundación Progreso y Salud y celebrada en Sevilla el 20 de marzo.

HUNT, K., y ANNANDALE, E. (1999): «Relocating Gender and Morbidity: Examining Men's and Women's Health in Contemporary Western Societies. Introduction to Special Issue on Gender and Health», Social Science \& Medicine, 48: 1-5.

IDLER, E. L., y BENYAMINI, Y. (1997): «Self - rated health and mortality: a review of twenty-seven community studies», J. Health Soc. Behav., 38: 21-37.

INSTITUTO DE ESTADíSTICA DE ANDALUCÍA (2002): Inmigración Extranjera en Andalucía, Sevilla.

INSTITUTO NACIONAL DE ESTADÍSTICA: www.ine.es/inebase

IZQUIERDO, J., y otros (1996): La desigualdad de las mujeres en el uso del tiempo, Instituto de la Mujer, Madrid.

JIMÉNEZ, C. (2002): Interculturalismo, ponencia presentada en el Foro sobre Inmigración organizado por la Asociación Pro-Derechos Humanos de Andalucía y celebrado en Cádiz los días 7 y 8 de junio.

JULIANO, D. (1992): El juego de las astucias, Cuadernos Inacabados 11, Editorial Horas y Horas, Madrid. 
JULIANO, D. (1993): «Las pobres mujeres del mundo pobre», en Sistemas de Género y Construcción (Deconstrucción) de la Desigualdad, Díez y Maqueira (coords.), VI Congreso de Antropología, Tenerife.

KARASEK, R., y THEORELL, T. (1990): Healthy Work: Stress, Productivity, and the Reconstruction of Working Life, Basic Books, New York.

KESSLER, R. C., y McLEOD, J. D. (1985): «Social Support and Mental Health in Community Samples», en Social Support and Health, Cohen y Syme (eds.), Academic Press, Orlando.

KLUCKHOHN, F., y STRODTBECK, F. (1961): Variations in Value Orientations, Row Peterson, Evanston.

LEVINTON, N. (2002): Género y salud mental. Dependencia amorosa y violencia de género, en Seminario del Programa de Formación Continuada del Hospital Universitario Virgen Macarena de Sevilla, celebrado el 7 de junio.

Ley Orgánica 4/2000, de 11 de enero, sobre Derechos y Libertades de los Extranjeros en España y su Integración Social, con la incorporación de la reforma operada por la LO 8/2000, de 22 de diciembre.

MAALOUF, A. (1999): Identidades asesinas, Alianza Editorial, Madrid.

MARMOT, M., et al. (1999): «Health and the Psychosocial Environment at Work», en Social Determinants of Health, M. Marmot y R. Wilkinson (eds.), Oxford University Press, New York.

MARQUÉS DÍEZ, B. (2000): Las dificultades psicológicas de adaptación de los trabajadores inmigrantes: informe sobre investigaciones y experiencias en España y Alemania, Confederación de Empresarios de Aragón.

MARTÍN, E. (1999): Procesos migratorios y relaciones interétnicas en Andalucía: una reflexión sobre el caso del Poniente almeriense desde la Antropología Social, Junta de Andalucía, Sevilla.

MÉDICOS DEL MUNDO - ANDALUCÍA (2002): Programa de Atención Sociosanitaria a Inmigrantes. Memoria Anual 2001, Sevilla.

MEILLASSOUX, C. (1987): Mujeres, graneros y capitales, Siglo Veintiuno Editores, México.

MINISTERIO DEL INTERIOR (2000) (www.mir.es/dgei): Anuario Estadístico de Extranjería, Delegación del Gobierno para la Inmigración y Extranjería.

MOHANTY, C., et al. (1990): Third World Women and the Politics of Feminism, Indiana University Press, Bloomington.

MONTERO-PIÑAR, I. (1999): «Factores de riesgo de depresión en las mujeres», en Trastornos depresivos en la mujer, C. Leal (coord.), Masson, Barcelona.

MORENO, L. (2000): Ciudadanos precarios. La última red de protección social, Ariel Sociología, Barcelona.

NAROTZKY, S. (1988): Trabajar en familia. Mujeres, hogares y talleres, Edicions Alfons el Magnànim, Institutió Valenciana d'Estudis i Investigació, Valencia.

NASH, M., y MARRE, D. (2001): Multiculturalismos y género. Un estudio interdisciplinar, Edicions Bellaterra, Barcelona.

OKPAKU, S. O. (1998): Clinical Methods in Transcultural Psychiatry, American Psychiatry Press, Washington.

OSO, L. (1998): La migración hacia España de mujeres jefas de hogar, Instituto de la Mujer, Madrid.

PORTES, A. (1983): «Modes of Strutural Incorporation \& Present Theories of Labor Inmigration: Theory and Research on International Population Movements», en Global Trends in Migrations, M. Kritz (ed.), Centre for Migration Studies, Staten Island, NY.

PROVANSAL, D. (1993): Migraciones, segregación y racismo, en Actas del VI Congreso de Antropología, Tenerife. 
REGIDOR, E., y cols. (2002): “Comparing Social Inequalities in Health in Spain: 1987 and 1995/1997», Soc. Sci. Med., 54 (9): 1323-32.

RODRÍGUEZ, A. (2002): La política económica y su repercusión en las mujeres, presentado en el Foro Social «Otro Mundo es Posible», celebrado en Sevilla en junio.

ROHLFS, I.; BORRELL, C., y FONSECA, M. do C. (2000): «Género, desigualdades y salud pública: conocimientos y desconocimientos», Gaceta Sanitaria, 14:60-71.

ROHLFS, I., y cols. (2001): «Influencia del trabajo dentro y fuera de casa sobre el estado de salud percibido de las mujeres», Medicina Clínica, 180: 566-571.

SEMFYC: La atención al inmigrante: del aluvión a la solución razonable (www.semfyc.es).

SENNETT, R. (2000): La corrosión del carácter, Anagrama, Barcelona.

SHILLING, R. S. F. (1989): «Health Protection and Promotion at Work», Br. J. Indust. Med., 46: 683-688.

SIEGRIST, J. (1996): «Adverse Health Effects of High-Effort / Low Reward Conditions», J. Occupat. H. Psychol., 1: 27-41.

SOS RACISMO: Informe anual 1999 sobre el racismo en el Estado español, Icaria Editorial, Barcelona.

STANSFELD, S. A., et al. (1998): «Types of Social Support as Predictors of Psychiatrist Morbidity in a Cohort of British Civil Servants (Whitehall II Study)», Psychol. Med., 28: 881-892.

STIGLITZ, J. E. (2002): El malestar en la globalización, Editorial Taurus, Madrid.

TÁVORA, A. (2000): ¿Por qué enferman las mujeres?, Taller de Discusión presentado en la Reunión de Equipos de Salud Mental de Andalucía celebrada en Granada, noviembre.

TSENG, W. S., y STRELTZER, J. (2002): Culture and Psychotherapy. A Guide to Clinical Practice, American Psychiatry Press (www.appi.org).

UGALDE, A. (1997): «Salud e inmigración económica del Tercer Mundo en España», en Inmigración, salud y políticas sociales, O. Solas y A. Ugalde (eds.), Escuela Andaluza de Salud Pública, Serie Monografías, n. ${ }^{13}$, Granada.

ULLOA, M. (2002): Reflexiones sobre el servicio doméstico, presentado en el Foro sobre Inmigración organizado por la Asociación Pro-Derechos Humanos de Andalucía y celebrado en Cádiz los días 7 y 8 de junio.

VARIOS AUTORES (1996): Tiempo social contra reloj. Las mujeres y la transformación en los usos del tiempo, Instituto de la Mujer, Madrid.

VARIOS AUTORES (2001): La situación social de las mujeres en Andalucía 1990-2000, Estudios 16, Instituto Andaluz de la Mujer, Sevilla.

WHITE, P. (1998): «Urban Life and Social Stress», en The New Europe: Economy, Society and Environment, D. Pinder (ed.), Wiley, Chichester.

WHITTLE, K. L., e INBORN, M. C. (2001): «Rethinking Difference: A Feminist Reframing of Gender/Race/Class for the Improvement of Women's Health Research», International Journal of Health Services, 31 (1): 147-165.

WHO (2001): The World Health Report 2001. Mental Health: New Understanding, New Hope, World Health Organisation (www.who.int/whr/2001). 


\section{ABSTRACT}

Most of the immigrant women that live in Sevilla work in Domestic Services. Their health complaints in emergency rooms and in family doctor consultations, following the impression of health workers, tell us more than about a syndrome, they include all their life story. Organisation of Health and Social Services for a medium - long term demand from professionals a look that integrate migrants movements, new labour relations in globalisation era, gender health relationships, and culture, that goes across everything. A professional look that try to understand, looking for diminishing avoidable inequalities in health.

Key words: Working Women, Immigrants, Health. 